\title{
Technique
}

\section{A technique of intestinal intubation with the fiberoptic endoscope}

\author{
REED T. KELLER From Case Western Reserve \\ University School of Medicine, Department of \\ Medicine, University Hospitals of Cleveland, Ohio
}

Prolonged obstruction of a gastric outlet can be a serious complication of gastric resection. Nutritional support may be necessary until the stoma functions properly. Intubation of the intestine with a feeding tube, even with fluoroscopic control, may prove impossible and intravenous feeding has both difficulties and risks.

This report describes the use of the fiberoptic endoscope to facilitate the positioning of an intestinal tube through an obstructed stoma. The same technique could be used for duodenal intubation in patients with gastric atony or pyloric narrowing.

\section{Case Report}

A 64-year-old male with rheumatoid arthritis was admitted with a massive upper gastrointestinal haemorrhage. Because of persistent bleeding, emergency surgery was performed and revealed a duodenal ulcer and a tear of the mucosa at the gastroesophageal junction. The gastroesophageal tear was repaired and a iso-peristaltic Billroth II gastrectomy with vagotomy was performed. The postoperative course was complicated by persistent obstruction of the gastric outlet. Intravenous feeding was instituted, but was discontinued after eight days when Gramnegative sepsis occurred. An upper gastrointestinal series of radiographs on the fourteenth postoperative day demonstrated no filling of the efferent loop. Fiberoptic gastroscopy revealed a patent afferent stoma; the efferent stoma appeared to be almost totally obstructed by oedematous jejunal mucosa surrounding a jejunal ulcer. However, the distal tip of the endoscope could be manipulated through the stoma with some difficulty. Repeated attempts at fluoroscopic placing of an Abbott-Rawson feeding tube in the efferent loop were unsuccessful. Using the tech-

Please request reprints from: Reed T. Keller, MD, Department of Medicine-University Hospitals of Cleveland, 2065 Adelbert Road, Cleveland, Ohio.

Received for publication 7 November 1972. nique described below, a Miller-Abbott tube was successfully placed in the efferent jejunal loop with the aid of a fiberoptic endoscope. High-caloric tube feedings were maintained until the patient's death from progressive pneumonia and pancreatitis.

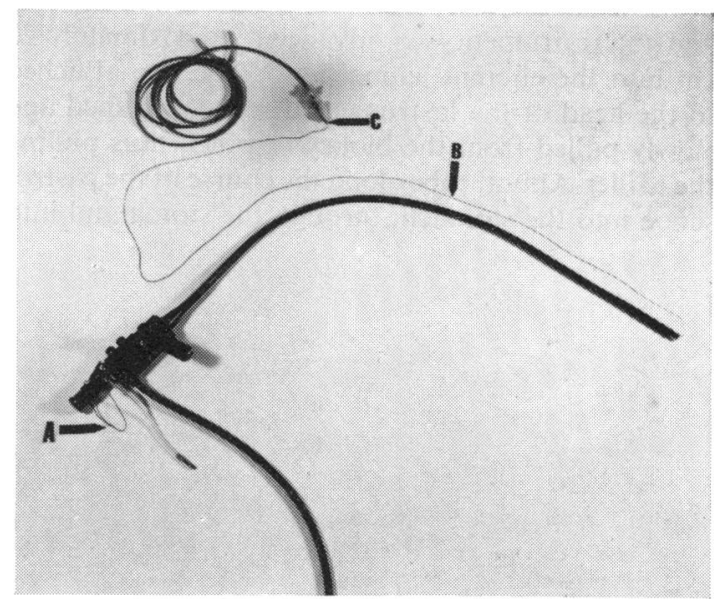

Fig. 1 Before passing the endoscope the line is tied to the eyepiece and passed through the biopsy channel (A), brought back along the outside of the endoscope (B), and tied to the metal tip of the Miller-Abbott tube (C).

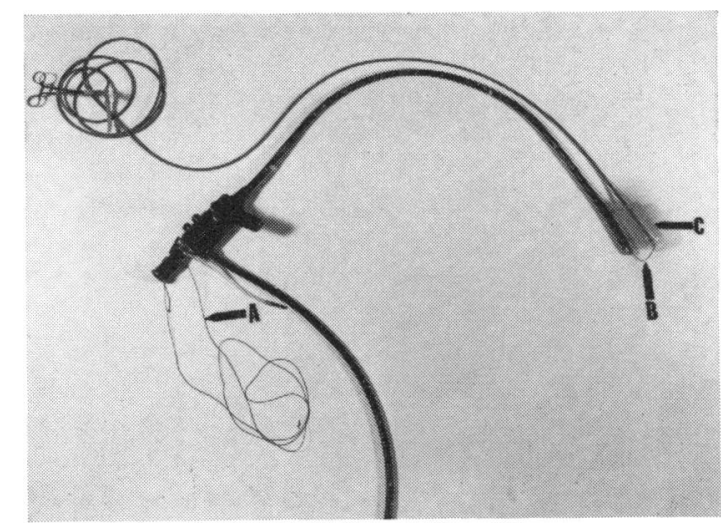

Fig. 2 With the distal end of the instrument through the stoma, the line is pulled from the biopsy channel (A), thus pulling the Miller-Abbott tube to the end of the endoscope (B). The bag is then inflated (C), and the endoscope removed. 


\section{Technique}

One end of a 10-foot piece of 25-pound test braided nylon fishing line was tied to the eyepiece of the ACMI fiberoptic poly-directional Panendoscope. The other end was passed through the biopsy channel using the biopsy forceps to grasp and direct the line. The line was then pulled from the distal end and brought back along the outside of the instrument; liberally applied surgical lubricant caused the line to adhere slightly to the endoscope. The free end of the line was then tied to the metal basket of a MillerAbbott tube (Fig. 1). The endoscope was then passed in the usual manner, the efferent stoma was entered, and the instrument was advanced approximately 20 $\mathrm{cm}$ into the efferent jejunal loop. The line attached to the head of the instrument was then grasped and slowly pulled from the biopsy channel, thus pulling the Miller-Abbott tube along the course of the gastroscope into the stomach, through the stoma and into the efferent loop. When the basket of the MillerAbbott tube reaches the distal tip of the instrument, the line can no longer be pulled. The bag of the MillerAbbott tube was fully inflated with air to anchor it in the jejunum (Fig. 2). The gastroscope was then slowly removed, allowing the line to slip through the biopsy channel. When the endoscope had been completely removed, the line was cut and tied to the Miller-Abbott tube. The bag was then deflated and the tube secured at the mouth with tape. Tube feedings were then instituted.

Attempts to pass an Abbott-Rawson tube in a similar fashion have been unsuccessful because the tube lacks an inflatable bag which is necessary to anchor the tube temporarily. Otherwise, the tube adheres to the endoscope and is removed inadvertently with the endoscope.

The author is grateful to the Cleveland Veterans' Administration Hospital for the photographs. 